\title{
Mix-Design and Control of Exposure Class of Durable Concrete
}

\author{
Peter Paulini \\ Material Technology Unit, University of Innsbruck, Austria \\ Corresponding author: peter.paulini@aon.at
}

\begin{abstract}
Durability requirements for concrete are determined by climatic, chemical and mechanical effects, depending on the climatic location and intended use of structural parts. The European standard EN 206-1 defines different types of exposure classes and specifies requirements for concrete, such as type and minimum quantity of cement, maximum w/c ratio, minimum compressive strength or minimum entrained air pore content. Additional characteristics required for a higher workability often lead to contradictory situations in practice and cannot be satisfactorily resolved by varying the mix design of the concrete. Based on a real life scenario where a C35/45 XD3 concrete had been used in the construction of a multistorey car park, which presented serious cracking after only a short service time, this paper proposes a number of methods designed to assess the quality and durability of concrete. The proof of exposure classes at the hardened building concrete is not regulated in existing national standards. Therefore, in many court disputes it is difficult to prove the quality of the hardened concrete because of lacking regulations. However, it is possible to verify the w/c-ratio using the capillary porosity. The basic relationships between cement content, w/c-ratio and capillary porosity are derived and discussed. Higher proportions of cement, which are necessary to achieve a better workability or higher strength, result in a reduced modulus of elasticity and increased shrinkage as well as a higher risk of cracking. The associated increase of capillary pore volume and of permeability reduces the service life of concrete. The determination of performance-based concrete properties such as permeability, chloride migration or electrical conductivity further complements the assessment of concrete durability. These properties can be used for estimating the resistance of the capillary pore structure to specific damage mechanisms.
\end{abstract}

\section{Introduction}

The notion of sustainability in modern economics totally changed the understanding of durability of materials in the construction sector. Typically, reinforced concrete (RC), the most prominent construction material, is a composite material consisting of cement paste as the binder, natural stone as the aggregate and steel as the reinforcement. The pore structure, which is of prime importance in this discussion, could be qualified as its fourth component. The pores can either be partly or fully saturated with water or, predominantly near the surface areas, be dry and filled with air. In most natural stones the proportion of pores is very small, below $1 \%$ in volume, and often not joined up in a capillary system. Therefore, only hardened cement paste (HCP) has a continuous capillary pore structure in which electrical, chemical and physical damage processes mainly can take place. As we know, monuments built in natural stone have survived centuries bearing witness to ancient civilisations. If we were to consider concrete as a binary composite material consisting of durable natural stone and a capillary porous matrix of $\mathrm{CSH}$-gel in which the potential for any damage were contained, a law of durability could be drawn up by a simple pragmatic logic: "reducing the proportion of binder to a minimum while maintaining workability and achieving the degree of strength required". This requirement not only has a technical but also a strong ecological and economic aspect.

Furthermore, however, in RC structures, the reinforcing material must also be resistant to corrosion. Corrosion-inducing processes typically start at the surfaces of a structural element, e.g. if attacked by saline solutions. Steel is a meta-stable material which oxidizes in the presence of water and oxygen to form iron oxide. In the alkaline environment of a saturated concrete pore water where $12<\mathrm{pH}<13.5$, normal oxygen corrosion is prevented by alkali passivation. Only in areas near to the surface areas of structural members portlandite $\mathrm{Ca}(\mathrm{OH})_{2}$ can carbonate into insoluble $\mathrm{CaCO}_{3}$ and the passivation is lost. This is a slow process requiring the presence of $\mathrm{CO}_{2}$ and water, i.e. alternating dry and humid conditions. National standards state a required cover concrete in order to minimise corrosion resistance of reinforcement. The speed of progression of carbonation or chloride penetration is determined by the capillary pore structure 
Table 1, Austrian concrete production grades acc. to ON B 4710-1 NAD $10+16$ [18]

\begin{tabular}{|l|l|c|c|c|}
\hline Type & \multicolumn{1}{|c|}{ Exposure Classes } & $\begin{array}{c}\text { wo/b } \\
\text { max. }\end{array}$ & $\begin{array}{c}\text { entrained } \\
\text { Air } \%-\mathbf{~}\end{array}$ & $\begin{array}{c}\text { min. Binder } \\
\mathbf{k g} / \mathbf{m}^{\mathbf{3}}\end{array}$ \\
\hline B1 & XC3 & 0.60 & & 280 \\
B2 & XC3/XD2/XF1/XA1L & 0.55 & & 300 \\
B3 & XC3/XD2/XF3/XA1L & 0.55 & $2.5-5.0$ & 300 \\
B4 & XC4/XD2/XF1/XA1L & 0.50 & & 300 \\
B5 & XC4/XD2/XF2/XA1L & 0.50 & $2.5-5.0$ & 320 \\
B6 & XC4/XD2/XF3/XA2L/XA2T & 0.45 & $2.5-5.0$ & 360 \\
B7 & XC4/XD3/XF4/XA1L & 0.45 & $4.0-8.0$ & 340 \\
B8 & XC3/UB1 & 0.60 & & 280 \\
B9 & XC3/UB2 & 0.60 & & 280 \\
B10 & XC3/XD2/XF1/XA1L & 0.55 & & 300 \\
B11 & XC3/XD2/XF1/XA1 & 0.55 & & 280 \\
B12 & XC3/XD2/XF1/XA1L & 0.50 & & 300 \\
\hline
\end{tabular}

and defines the time periods of carrying out repairs on the structure.

Most national and international standards stipulate durability requirements by defining exposure classes. For each class, the type of chemical, climatic or mechanical attack is stated and subdivided into 3 to 4 levels of intensity. Exposure classes are based on a particular mix design and define requirements on the following parameters:

- cement/binder type with minimum content in some standards

- use of supplementary cementitious materials (fly ash, blast furnace slag, silica fume)

- maximum allowed w/c ratio, decreasing with higher level of attack

- minimum grade of concrete in some standards minimum concrete cover

Progressively lowering the w/c-ratio for higher levels of attack reduces the capillary pore volume in HCP. However, the specification of minimum cement content or minimum concrete strength counteracts this intended result and leads to the erroneous assumption that increasing the binder content would lead to higher durability. Reinforcement bars in RC structures are passivated at a minimum binder content of about 280$300 \mathrm{~kg} / \mathrm{m}^{3}$ (EC2 NAD: C25/30 - C30/37). Higher contents of binder material are only required for certain classes of concrete strength or for improved workability and are opposed to increased durability. Some provisions in national annexes of EN 206 lead to situations where compliance with elevated exposure classes causes a reduction in durability, as shown in the example below.

The correct proportion of binder material in a concrete mix is the result of a process of optimization within a four-dimensional matrix of strength workability - durability and price. In our further discussion we concentrate primarily on the one material parameter - pore volume - which is of utmost significance for all four dimensions. For optimal strength and durability, pore volume should be minimized, while sometimes in practice it is maximized for better workability.

\section{Multi-storey Car Park - Court Case}

Leoben city shopping mall (LCS) including a two-storey car park was built in 2006. After having been in service for only five years severe crack damage was discovered in the floor slabs of the parking decks. The weight bearing structure consists of $35 \mathrm{~cm}$ thick concrete slabs, point supported and also based on the outer walls of the building. The concrete slabs were treated with a $3 \mathrm{~mm}$ epoxy surface cover providing protection against deicing humidity and carbonation. Surface water is drained through welded flat plastic drains connected to vertical guttering.

The engineer had opted for a C35/45 XD3 F3 type concrete for the RC structure. The type of concrete used in the deck slabs was equivalent to $\mathrm{C} 35 / 45 \mathrm{~B} 7$ according to ON B 4710-1 (Tab.1) [18]. This type of concrete fulfils requirements of exposure class XD3 and others. The conformity of concrete properties such as compressive strength (>53 MPa), air content $(6.7 \%)$ and flow (F450 mm) was tested during building. The binder consisted of $410 \mathrm{~kg} / \mathrm{m}^{3}$ cement CEM II-AS 42.5 and of $50 \mathrm{~kg} / \mathrm{m}^{3}$ supplementary cementitious materials. Initial testing for this type of concrete was based on a batch water of $189 \mathrm{~kg} / \mathrm{m}^{3}$. This concrete should contain a capillary pore volume of

$V_{c p}=(189-0.24 \cdot 450) / 10=8.1 \%-\mathrm{Vol}$

A court expertise confirmed that the prescribed quality of concrete C35/45 XD3 had been used and attributed the crack damage to insufficient steel reinforcement and to temperature-induced confining tensile stresses. Our 
Table 2. Test data of five year old LCS concrete

\begin{tabular}{|l|c|c|c|c|}
\hline \multicolumn{1}{|c|}{ Concrete Properties } & Dimension & \multicolumn{2}{c|}{ own tests } & $2^{\text {nd }}$ Court expert \\
\hline & & near surface & core & \\
\hline water saturated density & $\mathrm{kg} / \mathrm{m}^{3}$ & 2268 & 2298 & 2202 \\
\hline capillary dry density & $\mathrm{kg} / \mathrm{m}^{3}$ & 2020 & 2073 & \\
\hline mean total porosity & $\%-\mathrm{V}$ & 24.8 & 22.5 & 20.98 \\
\hline entrained air pores & $\%-\mathrm{V}$ & -- & -- & 6.6 \\
\hline capillary porosity & $\%-\mathrm{V}$ & 17.8 & 15.5 & 14.38 \\
\hline mean mix water & $\mathrm{kg} / \mathrm{m}^{3}$ & -- & 234 & 239 \\
\hline mean w/b ratio calculated & -- & -- & 0.52 & 0.53 \\
\hline mean compressive strength & $\mathrm{MPa}$ & -- & -- & 52.8 \\
\hline splitting tensile strength & $\mathrm{MPa}$ & -- & 3.09 & -- \\
\hline surface pull tensile strength & $\mathrm{MPa}$ & -- & -- & 3.1 \\
\hline Young modulus & $\mathrm{GPa}$ & -- & -- & 23 \\
\hline longitudinal US velocity & $\mathrm{m} / \mathrm{sec}$ & -- & 4485 & -- \\
\hline rebound ratio microhardness & -- & -- & 0.505 & -- \\
\hline coefficient air permeability dry spec. & $\mathrm{m}{ }^{2}$ & $3.33 \mathrm{E}-16$ & $2.47 \mathrm{E}-16$ & -- \\
\hline mean shrinkage accelerated $50{ }^{\circ} \mathrm{C}$ & -- & -- & $7.38 \mathrm{E}-04$ & -- \\
\hline
\end{tabular}

own testing of core samples taken from the slabs caused doubts on the concrete quality XD3 and we concluded that the cracks were caused by shrinkage stress. This assessment was then confirmed in a second court opinion which also found a very low static Young module. Table 2 summarizes the concrete properties described in both expert reports. Quite obviously, the capillary pore volume of the tested concrete, obtained in both reports, was far above the expected volume of $8.1 \%$-Vol.

Technical arguments presented to a court should always be based on the state of technology. However, national standards rarely set out criteria for verifying the exposure class of a hardened concrete structure in the instance of damage. Proof of the w/b-ratio in a hardened concrete can best be performed determining the capillary pore content. Furthermore performance-based specifications enable us to establish a durability class ranking. This approach is presented in detail in the following chapters.

\section{Capillary Pore Volume}

The water consumption of cement clinker phases is known from their stoichiometric reaction equations. In the chemically bonded state water loses volume and leads to a chemical shrinkage. For individual clinker phases the water demand can be expressed relatively to mass $\left(f_{w m}\right)$ or to volume $\left(f_{w v}\right)$ [1]. The same is possible for the chemical shrinkage factor $\left(f_{s}\right)$. With the proportions of Portland cement clinker these factors become (Eq. 1-3) [1]

$f_{w v}=\frac{V_{w}}{V_{c}}=0.7422 \cdot\left[\mathrm{C}_{3} \mathrm{~S}\right]+0.6874 \cdot\left[\mathrm{C}_{2} \mathrm{~S}\right]+1.2023 \cdot\left[\mathrm{C}_{3} \mathrm{~A}\right]+\ldots$

$+1.3754 \cdot[\mathrm{C} 4 \mathrm{AF}]$ $f_{w m}=\frac{M_{w}}{M_{c}}=0.2367 \cdot\left[\mathrm{C}_{3} \mathrm{~S}\right]+0.2092 \cdot\left[\mathrm{C}_{2} \mathrm{~S}\right]+0.4000 \cdot\left[\mathrm{C}_{3} \mathrm{~A}\right]+\ldots$ $+0.3919 \cdot[\mathrm{C} 4 \mathrm{AF}]$

$f_{s}=\frac{V_{h y}-V_{o}}{V_{o}}=-0.2243 \cdot[\mathrm{C} 3 \mathrm{~S}]-0.1909 \cdot[\mathrm{C} 2 \mathrm{~S}]+\cdots$ $+0.4455 \cdot[\mathrm{C} 3 \mathrm{~A}]+0.307 \cdot[\mathrm{C} 4 \mathrm{AF}]$

Therein $M_{c}$ and $V_{c}$ denote mass and volume of cement and the values in brackets represent the mass proportions of clinker phases. The ratio of the two factors $f_{w v}$ and $f_{w m}$ is the density ratio of cement to water.

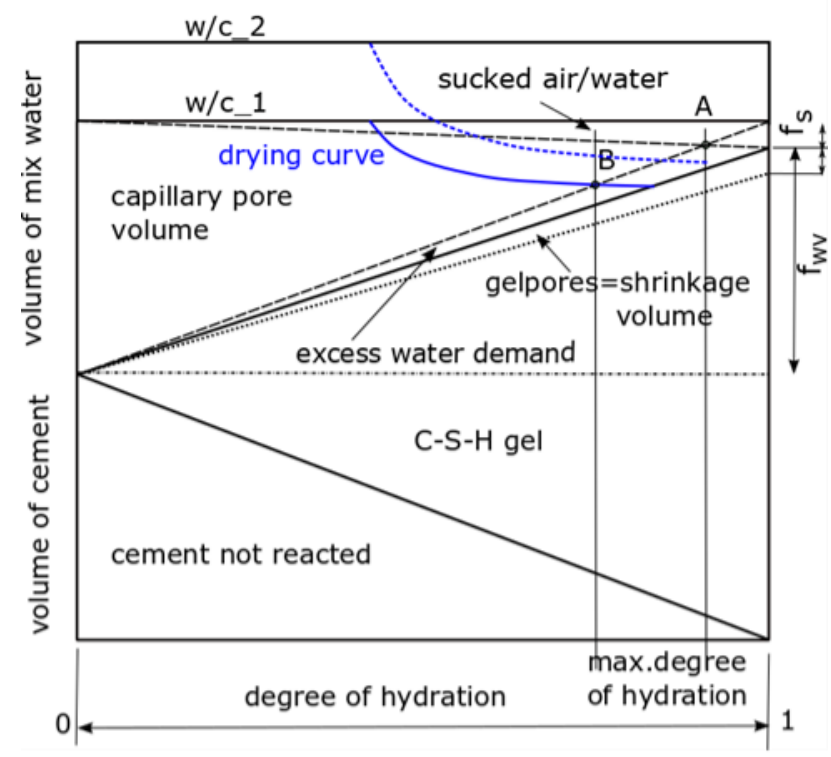

Fig.1 Linear hydration model of capillary volume in CSH-paste [1] 
$\frac{f_{w v}}{f_{w m}}=\frac{\rho_{c}}{\rho_{w}}$

For the various types of cement, the water demand factors change only slightly and are in the range of $0.80<f_{w v}<0.83$ and $0.24<f_{w m}<0.26$ respectively $[1,2]$. In the literature, mass based water demand values of 0.23 0.25 are mentioned [3, 4]. In this case a value of 0.24 will be assumed for $f_{w m}$. Based on a linear hydration reaction model, the volumes in $\mathrm{C}-\mathrm{S}-\mathrm{H}$-gel are shown in Figure 1. In practice, the above-mentioned factors for unknown stoichiometric reactions of supplementary cement additions vary considerably and should be measured.

Assuming a linear relationship with the degree of hydration $\alpha$, the capillary pore volume $V_{c p}$ in concrete becomes (Eq.5)

$V_{c p}=\frac{c}{\rho_{w}} \cdot\left(w c-\alpha \cdot f_{w m}\right)=\frac{c}{1000} \cdot(w c-\alpha \cdot 0.24)$

and the w/c-ratio for a given quantity of cement $C$ can be expressed as in (Eq. 6)

$w c=\frac{V_{c p} \cdot \rho_{w}}{C}+f_{w m} \cdot \alpha=\frac{V_{c p} \cdot 1000}{C}+0.24 \cdot \alpha$

Under ambient air conditions the CSH-gel needs twice the chemical shrinkage volume to achieve full hydration (Fig.1). Therefore, the minimum required w/c-ratio to reach full hydration is appr. $0.24+2 \cdot 0.065=0.37$. A low w/c-ratio and climatic conditions where pore water evaporates can considerably reduce the maximum degree of hydration, especially in regions near to the concrete surfaces. The capillary suction reduces the available hydration water in near-surface regions of the structure causing higher pore volumes in those areas. Typically climatic, physical and chemical attacks are strongest at the member surface which is why durability in these areas is always lower than in core regions. Therefore any assessment of concrete quality must necessarily distinguish between near surface and core concrete.

Assuming sound aggregates the total volume $V_{t}$ of the concrete specimen can be determined using Archimedes' principle according to national standards as difference between the vacuum saturated density $\rho_{s}$ and the dry density $\rho_{d}$ of $100^{\circ} \mathrm{C}$ dried concrete (Eq.7).

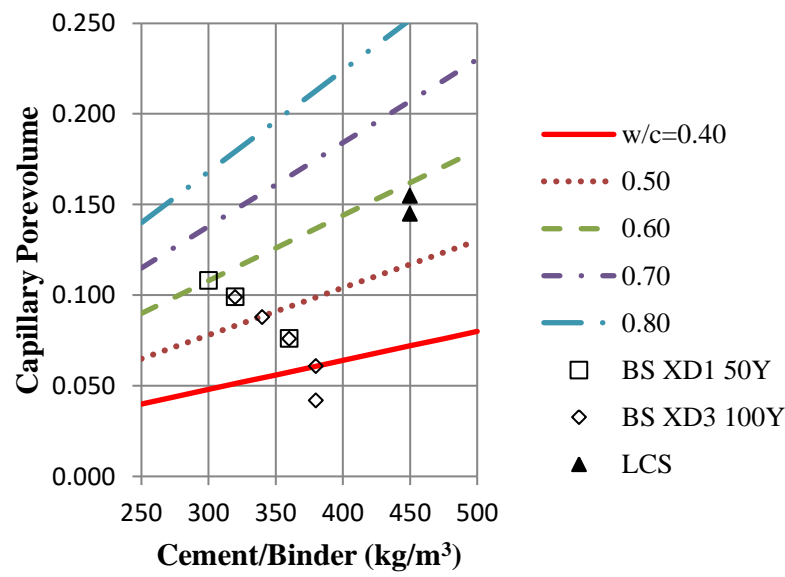

Therein $\rho_{w}$ denotes the water density. The apparent capillary pore volume $V_{c p}$ is then obtained by subtracting the entrained air pore volume $V_{\text {eap }}(0.01<\mathrm{d}<1 \mathrm{~mm})$ and the volume of compaction pores $V_{c o}(\mathrm{~d}>1 \mathrm{~mm})(\mathrm{Eq} .8)$. In order to calculate the ratio $w_{0} / c$ of the fresh concrete at mixing time using Eq.(6), the apparent capillary volume, established by density tests on the hardened concrete has to be reduced by the chemical shrinkage volume $V_{c s}$ (Eq.9). The degree of hydration $\alpha$ of the hardened concrete at testing time has to be chosen carefully. The remaining hydration potential of hardened concrete could also be tested on fine ground concrete powder using a buoyance weighing method [1].

$V_{t}=\frac{\rho_{s}-\rho_{d}}{\rho_{w}}$

$V_{c p}=V_{t}-V_{e a p}-V_{c o}$

$V_{c S}=\frac{C \cdot \alpha \cdot f_{S} \cdot f_{w m}}{1000}$

In Figures $2 \mathrm{a}, \mathrm{b}$ the capillary pore volume $\left(\mathrm{Eq} .5 \mathrm{~V}_{\mathrm{cp}}\right)$ and wc-ratio (Eq.6) are shown for concrete with different binder p0roportions for full hydration. These values are also numerically tabulated in Table 3. Some requirements of BS 8500-1 for XD1 and XD3 for a 50 and 100 year service life are also included in Fig. 2 a,b combined with the data obtained with the LCS concrete.

From Fig. 2a it is evident, that the requirements of a maximum w/c-ratio do not limit capillary pore volume. In Fig. $2 b$ isolines of constant capillary porosity for durability potential classes as proposed by BaroghelBouny [9] and shown in Tab.3 are plotted so as to place types of concrete in this field (Fig. 2). Quite obviously the LCS concrete falls outside the w/c limit and within the range of a medium to low durable concrete. If binder proportions are increased, the w/c-ratio needs to be decreased in order to limit capillary volume. Table 3 also shows a classification of concrete durability by limiting the binder proportions in several stages.

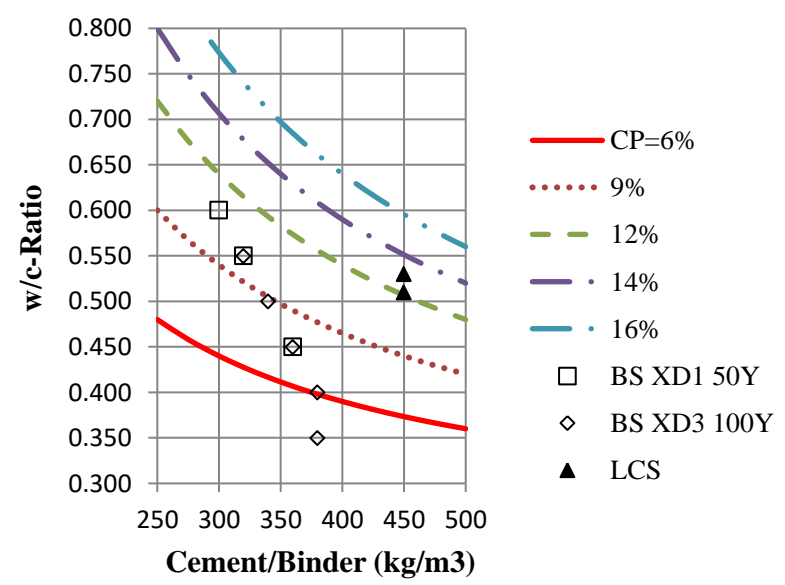

Fig 2 a,b Capillary pore volume and w/c ratio vs. binder of completely hydrated concrete and durability requirements of BS 8500-1 for XD1 and XD3 mixes vs. LCS concrete 
Table 3. Apparent capillary pore volume (Eq.5) of completely hydrated concrete $\left(f_{\mathrm{wm}}=0.24\right)$ and durability classes with limited binder content

\begin{tabular}{|c|c|c|c|c|c|c|c|}
\hline \multirow{3}{*}{$\begin{array}{c}\text { Binder in mix } \\
\left(\mathrm{kg} / \mathrm{m}^{3}\right) \\
\text { Durability class } \\
\end{array}$} & \multicolumn{7}{|c|}{ w/b-ratio } \\
\hline & 0.40 & 0.45 & 0.50 & 0.55 & 0.60 & 0.65 & 0.70 \\
\hline & \multicolumn{2}{|c|}{ very high } & \multicolumn{2}{|c|}{ high } & medium & low & very low \\
\hline 275 & $4.4 \%$ & $5.8 \%$ & $7.2 \%$ & $8.5 \%$ & $9.9 \%$ & $11.3 \%$ & $12.7 \%$ \\
\hline 300 & $4.8 \%$ & $6.3 \%$ & $7.8 \%$ & $9.3 \%$ & $10.8 \%$ & $12.3 \%$ & $13.8 \%$ \\
\hline 325 & $5.2 \%$ & $6.8 \%$ & $8.5 \%$ & $10.1 \%$ & $11.7 \%$ & $13.3 \%$ & $15.0 \%$ \\
\hline 350 & $5.6 \%$ & $7.4 \%$ & $9.1 \%$ & $10.9 \%$ & $12.6 \%$ & $14.4 \%$ & $16.1 \%$ \\
\hline 375 & $6.0 \%$ & $7.9 \%$ & $9.8 \%$ & $11.6 \%$ & $13.5 \%$ & $15.4 \%$ & $17.3 \%$ \\
\hline 400 & $6.4 \%$ & $8.4 \%$ & $10.4 \%$ & $12.4 \%$ & $14.4 \%$ & $16.4 \%$ & $18.4 \%$ \\
\hline 425 & $6.8 \%$ & $8.9 \%$ & $11.1 \%$ & $13.2 \%$ & $15.3 \%$ & $17.4 \%$ & $19.6 \%$ \\
\hline 450 & $7.2 \%$ & $9.5 \%$ & $11.7 \%$ & $14.0 \%$ & $16.2 \%$ & $18.5 \%$ & $20.7 \%$ \\
\hline 475 & $7.6 \%$ & $10.0 \%$ & $12.4 \%$ & $14.7 \%$ & $17.1 \%$ & $19.5 \%$ & $21.9 \%$ \\
\hline
\end{tabular}

\section{Concrete Durability Indicators}

Over the past two decades, more research has been done to increase the durability of concrete. Large infrastructures are often concrete structures designed for long service lives. Increased climatic, chemical and mechanical attack leads to higher demands on the quality of concrete. In addition to the description of durable concrete quality as defined in standards, performancebased test methods have been developed which are intended to enable both rapid classification of concrete grades and at the same time an assessment of their service life [5, 6]. Unfortunately, such methods have so far rarely been included in national standards because of the often still considerable scattering of measurement data.

Concrete durability is defined in relation to different types of damage resulting from specific transport or degradation mechanisms. Essentially, these relate to moisture and ion transport, electron conduction and gas transport. All these processes occur over longer time periods and become visible as structural damage only due to cumulative effects of repeated partial damage cycles. Test methods for assessing concrete damage are therefore time-consuming and have often been complemented by accelerated lab procedures. Such durability indicators enable us to correlate an accelerated damage process observed in the laboratory to one occurring in a real building [7-10]. A compilation and comparison of test methods for the durability of concrete can be found in $[13,14]$. Baroghel-Bouny proposed very meaningful durability indicators for various test methods in $[9,10]$. These data, complemented by the electrical conductivity data according to [11] and a proposal for maximum binder proportions are shown in Table 4 .

Usually test methods for determining accelerated transport characteristics are performed in the laboratory and require a reference state. Capillary dry concrete is used for testing gas permeability whereas diffusion and electrical conductivity tests are carried out on totally saturated concrete [11]. For on-site tests constant reference conditions cannot be guaranteed and they are always performed on partially saturated concrete with undefined humidity conditions. Therefore, on site tests show large scattering and are hardly suitable for a long termed quality assessment of concrete. According to the

Table 4. Proposed Durability Indicators [9] and [11] expanded by maximum Binder proportion

\begin{tabular}{|c|c|c|c|c|c|c|}
\hline Durability Potential & Dimension & $\begin{array}{c}\text { very } \\
\text { low }\end{array}$ & low & medium & high & $\begin{array}{c}\text { very } \\
\text { high }\end{array}$ \\
\hline Porosity accesible to water & $\%$-Volume & $>16$ & $14-16$ & $12-14$ & $9-12$ & $6-9$ \\
\hline Effective chloride diffusion coefficient & $10^{-12} \mathrm{~m}^{2} / \mathrm{sec}$ & $>8$ & $2-8$ & $1-2$ & $0.1-1$ & $<0.1$ \\
\hline Apparent chloride diffusion coefficient & $10^{-12} \mathrm{~m}^{2} / \mathrm{sec}$ & $>50$ & $10-50$ & $5-10$ & $1-5$ & $<1$ \\
\hline Apparent gas permeability & $10^{-18} \mathrm{~m}^{2}$ & $>1000$ & $300-1000$ & $100-300$ & $30-100$ & $<30$ \\
\hline $\begin{array}{c}\text { Intrinsic liquid water permeability } \\
10^{-18} \mathrm{~m}^{3}\end{array}$ & $>10$ & $1-10$ & $0.1-1$ & $0.01-0.1$ & $<0.01$ \\
\hline Initial Ca(OH)2 content & $\%$-Mass & $<10$ & $10-13$ & $13-20$ & $20-25$ & $>25$ \\
\hline Surface resistivity AASHTO T 358 & Ohm.m & $<120$ & $120-210$ & $210-370$ & $370-2540$ & $>2540$ \\
\hline maximum Binder proportion in mix & $\mathrm{kg} / \mathrm{m}^{3}$ & $>525$ & $<525$ & $<475$ & $<425$ & $<375$ \\
\hline
\end{tabular}


Swiss standard SIA 262/1 Annex E air permeability tests using the Torrent method may be performed with maximum concrete moisture of as much as $5.5 \%-\mathrm{M}$. This corresponds to a pore volume of more than $11 \%-\mathrm{V}$ based on a dry density of approximately $2.0 \mathrm{t} / \mathrm{m}^{3}$. Most concrete types with w/c-ratios of up to 0.55 would be fully saturated and would not allow any gas transport (Tab. 3). On the other hand, due to the suction effect of chemical shrinkage and evaporation, near-surface layers can only hydrate to lower degrees of hydration with higher pore volumes compared to core concrete. Nevertheless, on-site measurements of air permeability $k T$ taken by the Torrent method produce values far below those obtained on dry samples. Air permeability $k T$ may vary by a factor of 1000 in the saturation range $0<\mathrm{S}<1$ [12, Fig. 7].

It follows from the above that performance characteristics for the capillary pore system can only be reliably established under reference conditions of capillary dry or totally saturated concrete. The damage mechanisms underlying most concrete exposure classes are dependent only on the transportability of the capillary system. If one plots the mean values of the conductivity coefficients listed in Table 4 against air permeability, the log-log representation shows a good linear correlation (Fig. 3). However, the marginal values for the "very high" durability potentials have been eliminated in the plot due to the greater scattering of these time-consuming tests.

Assuming that the same capillary system is equally effective for each transport mechanism, the exponents would have to be exactly one. Despite the fact that class limits of the transport coefficients listed in Tab.4 are only roughly defined and are distributed in a variation range, the exponents of their mean values in the power law are close to 1 . The air permeability of LCS concrete in Fig. 3 falls in the range of "medium to low", which corresponds to the classification based on capillary porosity (Fig. 2b). Unfortunately, no chloride diffusion tests were performed during the damage assessment of LCS concrete, which would have led to a more accurate ranking for chloride resistance.

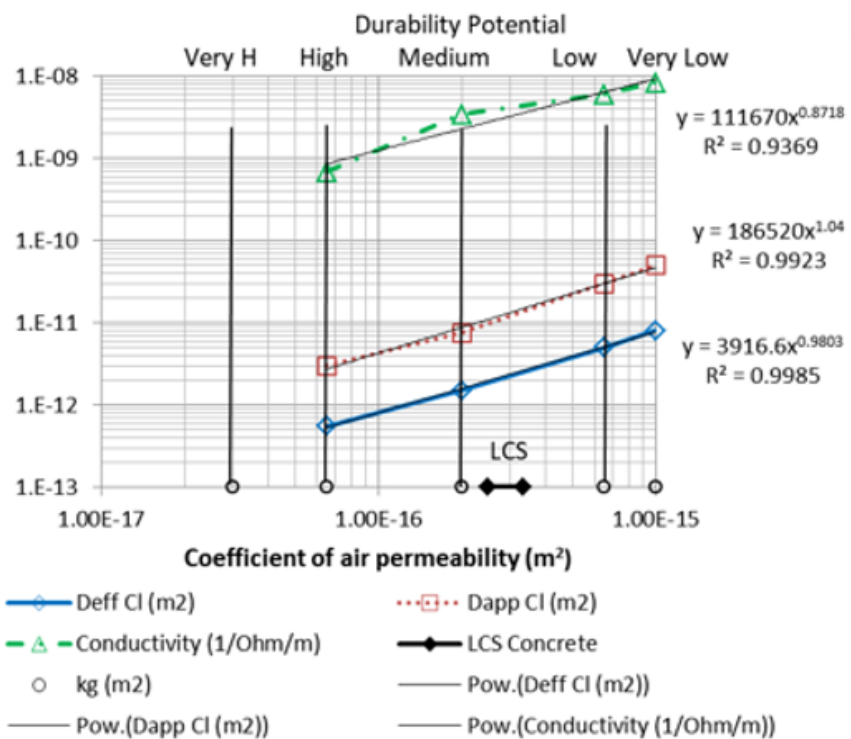

Fig.3. Mean transport coefficients of Tab. 4 vs. gas permeability
More sensitive and more reliable parameters for gas flow in porous materials have been described in [16] based on a power law. Transport laws based on power laws have been known for a long time and have been applied to the air permeability of concrete in [15]. A high correlation in concrete has also been found for coefficient of air permeability, carbonation depth and chloride ion diffusion in [17]. However, the type of cement and the usage of supplementary cementitious materials have a strong influence on the diffusion of chloride ions in concrete and may shift the relations given in Fig 3. Common understanding is that CEM I cements have a lower chloride binding capacity than mixed cements, especially using blast furnace slag. Our own tests have also shown low non steady state $\mathrm{Cl}$ migration coefficients $D_{\text {nssm }}$ using condensed silica fume [20].

The actual damage to the LCS parking decks was caused by $0.3-1.2 \mathrm{~mm}$ wide separation cracks through the entire concrete slab. Saline melting water penetrated the concrete slab causing an accumulation of chloride in the crack area. Clearly the severe cracking was due to the binder-rich concrete mix resulting in high shrinkage. Furthermore, the surprisingly low Young modulus of the concrete contributed significantly to the high shrinkage deformations. Initially, the stiffness of the concrete was misjudged on the basis of ultrasonic (US) measurements taken from water-saturated samples and was ultimately established at $23 \mathrm{GPa}$ by means of static tests on cylindrical cores. A more detailed analysis using US data on dry samples would also have yielded this result, as shown below.

The p-wave modulus $M$ and the dynamic E-modulus $E_{d y n}$ arise from Eq.(10) and (11)

$M=v_{L}^{2} \cdot \rho$

$E_{d y n}=M \cdot \frac{(1+v) \cdot(1-2 v)}{1-v}$

Ultrasonic waves propagate in concrete in the rigid composite material of HCP and rock aggregates. Waterfilled capillary pores are too small and are not captured by the wavelengths at frequencies of $50-250 \mathrm{kHz}$. While the correct assumption of Poisson's ratio is of paramount importance for the assessment of concrete stiffness (Eq.11). Establishing it on the basis of US shear waves is often difficult and inaccurate due to superimposed echoes. A simple and fast determination of the Poisson's ratio consists in using the rebound number $Q$ of rebound hammers (Schmidt Hammer, Micro-hardness Tester). Assuming a reduced rebound number $q_{r}$ Poisson's ratio is calculated in Eq.(12)

$v=\frac{1-q_{r}}{2}$

The reduction of the rebound number is particularly necessary when testing materials with high stiffness. The calibration of Schmidt hammers on a test block leads to rebound numbers of $Q=84 \%$, which must be reduced by $50 \%\left(q_{r}=0.42\right)$ for roughly the same moduli of elasticity of plunger and test block. By applying Eq.(12), 
Poisson's ratio of the calibration block becomes 0.29, falling well in the range for steel materials. If the stiffness differences of impactor and test material differ widely, the reduction has only a minor effect. Therefore, Micro-hardness testers with tungsten-carbide spheres as intender with E-moduli of 580-650 GPa are very suitable for concrete. For the LCS concrete, the mean rebound number of 0.505 (Tab. 2) results in a Poisson's ratio of 0.248 without reduction, and 0.26 with a reduced rebound number (as used in Table 5). The static modulus of elasticity of a porous concrete is calculated here as the mean value of a lower bound and an upper bound limit according to Eq.(13). The determination of the LCS static E-modulus from US data is shown in Tab.5. A detailed description for NDT determination of Poisson's ratio and relative modules will be given elsewhere

$$
E_{s t, m}=\frac{M}{4} \cdot\left(5 \cdot q_{r}-q_{r}^{2}\right)
$$

\section{Discussion}

The bundling of multiple exposure classes in Austrian standard ÖN B 4710-1 led to the situation that concrete plants in Austria normally only supply concrete classes listed in Tab. NAD 16 of this standard. Other specifications will only be delivered upon special order. The minimum cement content required by ÖN B 4710-1 for concrete grades complying with exposure classes XF are at least $20 \mathrm{~kg} / \mathrm{m}^{3}$ higher than that of other comparable national standards. If, in addition, a higher concrete strength class is requested, the required proportion of cement increases even more strongly and reaches quite unusually high levels. In the damage scenario described above, the typical characteristic compressive strength in the structure $(>42 \mathrm{MPa})$ was respected due to an equivalent binder content of 450 $\mathrm{kg} / \mathrm{m}^{3}$ in the mix, but at the same time the concrete presented an extremely low Young modulus and high shrinkage. Therefore, it can be argued that the unfortunate bundling of exposure classes in the aforementioned standard played a significant role in

Table 5. Ultrasonic data of LCS concrete and calculation of elastic modules acc. Eq.(11), (13)

\begin{tabular}{|c|c|c|c|c|c|c|c|}
\hline \multicolumn{1}{|c|}{ US-Longitudinal } & \multicolumn{4}{c|}{ Poisson's ratio= $\mathbf{0 . 2 6 0}$} \\
\hline Specimen & Length & dry Density & US Time_long & US Velocity_long & p-wave Module & E_dyn & E_st,m \\
\hline & $(\mathrm{mm})$ & $(\mathrm{kg} / \mathrm{m} 3)$ & $($ my_sec $)$ & $(\mathrm{m} / \mathrm{s})$ & $(\mathrm{GPa})$ & $(\mathrm{GPa})$ & $(\mathrm{GPa})$ \\
\hline $1.1 \mathrm{~m}$ & 121 & 1999 & 26.3 & 4600.8 & 42.31 & 34.58 & 24.01 \\
$1.2 \mathrm{~m}$ & 124 & 2147 & 28.3 & 4381.6 & 41.22 & 33.69 & 23.39 \\
$1.3 \mathrm{~m}$ & 134 & 2131 & 29.4 & 4557.8 & 44.27 & 36.18 & 25.12 \\
$1.4 \mathrm{~m}$ & 99 & 2004 & 22.9 & 4323.1 & 37.45 & 30.61 & 21.25 \\
$1.5 \mathrm{~m}$ & 122 & 2021 & 25.8 & 4728.7 & 45.19 & 36.93 & 25.65 \\
$2.2 \mathrm{~m}$ & 138 & 2037 & 30.3 & 4554.5 & 42.25 & 34.53 & 23.98 \\
$2.3 \mathrm{~m}$ & 134 & 2025 & 30.7 & 4364.8 & 38.58 & 31.53 & 21.89 \\
$2.4 \mathrm{~m}$ & 132 & 2155 & 30.3 & 4356.4 & 40.90 & 33.43 & 23.21 \\
$2.5 \mathrm{~m}$ & 134 & 2153 & 29.8 & 4496.6 & 43.53 & 35.58 & 24.70 \\
\hline Mean & & & & & $\mathbf{4 1 . 7 5}$ & $\mathbf{3 4 . 1 2}$ & $\mathbf{2 3 . 6 9}$ \\
\hline StdDev & & \multicolumn{7}{r|}{} & & & 2.39 & 1.95 & 1.36 \\
\hline
\end{tabular}

The crack damage in the LCS parking decks can be attributed to two main causes:

- $\quad$ the required quality of a concrete C35/45 XD3 as supplied to the site, did not respect the maximum permissible w/b-ratio of 0.45 . The w/b-ratio of the core concrete was about 0.53 , which meant that an increased proportion of capillary porosity was present in the concrete

- based on Austrian standard ÖN B 4710-1 the concrete B7 with a w/b-ratio of less than 0.45 complies exposure class XD3, it also needs to fulfil the requirements of exposure class XF4, for which an additional fraction of entrained air-pores of $4-8 \%-\mathrm{V}$ is needed. These air pores additions resulted in a binder-rich mix in a total pore content of $22-25 \% \mathrm{~V}$ for the LCS concrete. causing the severe crack damage in the LCS parking decks.

\section{Conclusions}

The methods to demonstrate compliance with exposure classes of concrete in cases of structural damage are generally not regulated in existing national standards and evidence in court cases can only be brought based on state-of-the-art procedures. One of these consists in demonstrating compliance with a given $\mathrm{w} / \mathrm{c}$ ratio based on the verification of the capillary porosity present in the concrete. So far, performance-based transport criteria have only rarely been adopted by standards to substitute durability of concrete and can best be used as complementary arguments.

In this paper, we discuss the fundamental relationships between concrete mixing data and capillary porosity and present the results graphically. Furthermore, we demonstrate the correlation between transport 
coefficients of major damage mechanisms (Durability Indicators) in concrete. They can be linearized by approximation in a log-log representation and divided into resistance classes. In addition to commonly accepted indicators, we proposed to use the binder content of the concrete mixture as an additional constraint.

High capillary pore content does not only accelerate damage mechanisms by increasing transport coefficients but also reduces concrete stiffness. Such types of concrete are vulnerable to shrinkage deformations and have a higher crack risk. The paper also presents NDT methods for determining realistic values for the static Young's modulus and Poisson's ratio of concrete.

\section{References}

1. P. Paulini, Zement-Kalk-Gips 41 Jg.(10), 525-531, (1988), https://bit.ly/2Xx11Bx

2. P. Paulini, N.V. Waubke, Bauingenieur, 65, 373379 (1990),

3. P.C Hewlett, F.M. Lea, Lea's Chemistry of Cenemt, Butterworth-Heinemann Ltd., (2004)

4. H.W.F. Taylor, Cement Chemistry, $2^{\text {nd }}$ Ed., Thomas Telford Publishing, (1997)

5. H, Beushausen, L.F. Luco, Performance-based specifications and control of concrete durability, RILEM TC 230-PSC Report, Springer, (2016)

6. J. Kropp J., H.K. Hilsdorf, Performance Criteria for Concrete Durability, RILEM Report 12, Taylor \& Francis, (1995)

7. L. Basheer, J. Kropp, J. Cleland, Cement and Concrete Composites, 15, 93-103, (2001)

8. T. Luping, L-O. Nilsson, P.A.M. Basheer, Resistance of concrete to chloride ingress, Testing and Modelling, Taylor \& Francis, (2012)

9. V. Baroghel-Bouny, Proc. 5th Int. Conf. Concrete under Severe Conditions Environment and Loading, CONSEC 07, 241 - 248, (2007)

10. V. Baroghel-Bouny, K. Kinomura, M. Thiery, S. Moscardelli, Cement and Concrete Composites, 33, 832-847, (2011)

11. AASHTO T 358-17, Standard Method of Test for Surface Resistivity Indication of Concrete's Ability to Resist Chloride Ion Penetration, (2017)

12. R. Torrent, F. Moro F., A. Jornet, RILEM Proceedings PRO, 89, Int. Workshop on performance-based specifications and control of concrete durability, Zagreb 2014, 489-498, (2013 $\mathrm{Ed})$

13. D. Bjegovic et al., Test methods for Concrete durability Indicators, Chapter 4 in [5] (2016)

14. K. Yang, S. Nanukuttan, W.J. McCarter, A. Long, P.A.M. Basheer, Revista ALCONPAT, 8 (3) 246263, (2018)

15. P. Paulini, F. Nasution, Proc. 5th Int. Conf. Concrete under Severe Conditions Environment and Loading, LCPC, Paris, 241-248, (2007)
16. P. Paulini, Transport in Porous Media, 128(1), 303 318, (2019)

17. Katpady, D.N., Hazehara, H., Soeda, M. et al., Int J Concr. Struct Mater (2018)

18. Austrian Standard ON B 4710-1: 2007, Beton Festlegung, Eigenschaften, Herstellung, Verwendung und Konformität - Teil 1

19. Swiss Standard SN 505 262/1 SIA 262/1:2013, Betonbau - Ergänzende Festlegungen

20. D. Moreau, Comparative study of air permeability and chloride ingress into concrete using the Nordtest method NT Build 492, Master Thesis, Univ. Innsbruck, (2017) 